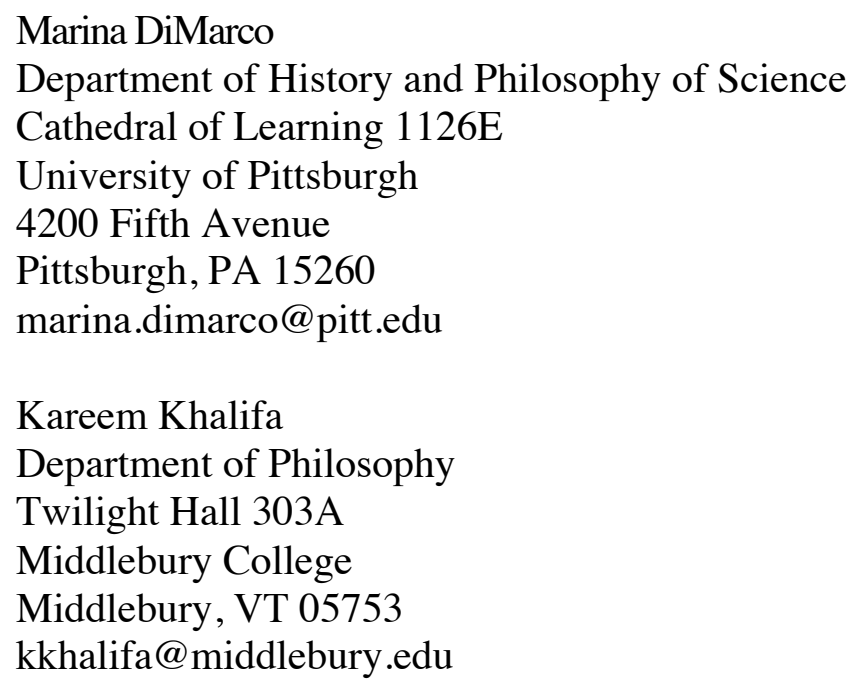

\title{
Inquiry Tickets: Values, Pursuit, and Underdetermination
}

\begin{abstract}
We offer a new account of the role of values in theory choice that captures a temporal dimension to the values themselves. We argue that non-epistemic values sometimes serve as "inquiry tickets," justifying scientists' pursuit of certain questions in the short run, while the answers to those questions mitigate transient underdetermination in the long run. Our account of inquiry tickets shows that the role of non-epistemic values need not be restricted to belief or acceptance in order to be relevant to hypothesis choice: the relevance of non-epistemic values to a particular cognitive attitude with respect to $h$ vary over time.
\end{abstract}

\section{Acknowledgements}

We are grateful to Kevin Elliott for his careful and insightful attention to this argument, and to audience members at PSA 2018 and members of the University of Pittsburgh HPS Works in Progress community for their feedback on earlier versions of the paper. 


\section{Introduction}

While the science-values relationship and the underdetermination of theory by evidence have been investigated independently, they also enjoy a history of symbiosis (e.g., Anderson 2004, Longino 1990, Nelson 1993). Beginning with the Quine-Duhem thesis, questions of underdetermination have arisen in conjunction with a reexamination of the privileged ideal of value-free science. More recently, philosophical projects addressing science and values have increasingly focused on temporary or "transient" underdetermination, as clear instances where values play an epistemic role (Biddle 2013, Brown 2013, Elliott 2011, Intemann 2005).

Interestingly, however, while transient underdetermination has clear temporal dimensions, the aforementioned scholarship on science and values has not accorded values similar temporal dimensions. In other words, while all agree that transient underdetermination behaves differently in the short- and long-term, values - whether epistemic or not - are generally assumed to play the same role over all timescales.

In this paper, we explore non-epistemic values' important temporal dimensions, by highlighting their role as “inquiry tickets.” In this capacity, they justify scientists' pursuit of certain questions in the short run, and the answers to those questions mitigate transient underdetermination in the long run. Because inquiry tickets only involve pursuit in the short run, the role of non-epistemic values need not be restricted to belief or acceptance in order to be relevant to hypothesis choice: the relevance of non-epistemic values to a particular cognitive attitude with respect to $h$ vary over time. In this way, we join others in highlighting the different relationships between non-epistemic values and various cognitive attitudes (!!! INVALID CITATION !!! ). Section 2 presents our account of inquiry tickets. Section 3 then discusses its implications for debates about science and values. While inquiry tickets are compatible with the 
ideal of value-free science, they replicate many science-values interactions thought incompatible with that ideal.

\section{What are Inquiry Tickets?}

Section 2.1 presents our account of inquiry tickets. Section 2.2 then illustrates how nonepistemic values can function as inquiry tickets with an example from biology.

\subsection{Framework}

As stated above, values function as inquiry tickets when they justify the pursuit of a question, where the answer to that question breaks an earlier tie between two competing hypotheses. More precisely, non-epistemic considerations' role in mitigating transient underdetermination must be understood as spanning three distinct stages of inquiry:

Transient Underdetermination: At time $t_{0}$, all of the epistemic considerations available at $t_{0}$ equally support competing hypotheses $h$ and $h^{*}$. Call the set of these considerations $E_{0}$.

Short Run: At time $t_{1}$, pursuing a question $Q$ is conducive to a set of goals $G$ adopted by some scientist $S$, and $Q$ is such that:

(1) If the pursuit of $Q$ were successful, then a currently unavailable epistemic consideration, $e$, would be made available, and

(2) If $e$ were true, it would, in conjunction with all of the members of $E_{0}$, support $h^{*}$ over $h$. 
Long Run: At time $t_{2}$, the pursuit of $Q$ succeeds. As a result, $e$ is available, and $h^{*}$ is better supported by the conjunction of $e$ and the members of $E_{0}$ than $h$ is. Hence, it is more rational to believe $h^{*}$ than it is to believe $h$.

We shall call any member of $G$ that plays this complex, intertemporal role an inquiry ticket. Our claim is that some inquiry tickets are non-epistemic (i.e. ethical, social, or political) values. To better motivate this thesis, we discuss each of these three stages in greater detail.

\subsubsection{Transient Underdetermination $\left(t_{0}\right)$}

The need for inquiry tickets arises in response to transient underdetermination. In general, in underdetermination, a given body of evidence fails to pick out a unique theory. This can be given weaker or stronger glosses. We have opted for a relatively modest gloss.

Because many early defenders of non-epistemic values in science (Anderson 1995, Longino 1990, 2002, Nelson 1990, 1993) borrowed heavily from (Duhem 1954), (Quine 1951), and (Kuhn 1977), they inherited a much bolder underdetermination thesis, namely:

Global Underdetermination Thesis: For any hypothesis $h$ and any set of epistemic considerations $e$, there is some other hypothesis $h^{*}$ that is as well supported as $h$ by $e .^{1}$

\footnotetext{
${ }^{1}$ The literature is not unanimous in characterizing these particular theorists as proponents of global underdetermination. We think that the figures cited above are at least ambiguous on this front. However, the paper's main argument does not hinge on these finer exegetical points.
} 
As many have argued, it is unclear whether global underdetermination is actually a feature of scientific practice, or merely a philosophical contrivance (!!! INVALID CITATION !!! ). In contrast, we only hold that some hypotheses are underdetermined, and only for a time, i.e.,

Transient Underdetermination Thesis (TUT): There exists some hypothesis $h$ and some time $t$, such that there is some other hypothesis $h^{*}$ that is as well supported as $h$ by all of the epistemic considerations available at $t$.

A brief terminological clarification: in its traditional slogan form, evidence underdetermines theories. We have opted for the phrase "epistemic considerations," to pinpoint the sticking point in debates about value-free science. Empirical evidence is one kind of epistemic consideration, but others include rules of reasoning that take evidence and background information as their inputs and probabilities of hypotheses as their outputs. Rules of reasoning include the canons of inductive and deductive inference, plus some prima facie plausible methodological maxims, e.g. "ceteris paribus, if $h$ correctly predicts some novel phenomenon $e$, then $\mathrm{P}(h \mid e)>\mathrm{P}(h)$." Some of these rules and maxims presumably invoke traditional theoretical virtues or "epistemic values" such as simplicity, scope, fruitfulness, fit with background beliefs, etc. At any rate, for our purposes, we can rather indiscriminately refer to all of these as "epistemic considerations" without loss.

\subsubsection{Short Run $\left(t_{1}\right)$}

As noted above, transient underdetermination highlights evidence and hypotheses' temporal dimensions, but non-epistemic values also require an analogous temporalization. We begin by 
examining the stage of inquiry after transient underdetermination but before its resolution — the "short run."

Scientists punch their inquiry tickets by adopting a future-oriented attitude in order to look beyond the transient underdetermination in the short run, so as to discover additional and decisive epistemic considerations in the long run. This future-oriented attitude is not belief; it is pursuit. We define pursuit as follows:

A person $S$ pursues a question $Q$ if and only if some of $S$ 's actions have the discovery of a correct answer to $Q$ as their goal.

While canonical discussions take theories to be the objects of pursuit (Laudan 1977, Whitt 1990), more recent discussions have broadened this to include phenomena, correlations, and technologies, among other things (!!! INVALID CITATION !!! ). We prefer to think of questions as the objects of all pursuits. Questions in turn can be about theories, phenomena, etc. Quite naturally, pursuits are successful just in case correct answers to the questions of interest are discovered.

In the context of transient underdetermination, non-epistemic considerations can justify certain kinds of pursuit that furnish tie-breaking epistemic considerations. To that end, we loosely follow (Seselja, Kosolosky, and Strasser 2012) in defining rational pursuit as follows:

It is rational for $S$ to pursue a question $Q$ if and only if pursuing $Q$ is conducive to $S$ 's set of goals $G$.

Now, our definition of pursuit builds one goal into $G$ automatically: if someone pursues a question, then the discovery of at least one correct answer to that question is one of her goals. However, this seems broadly epistemic in its orientation. Non-epistemic goals can also be in $G$. 
We provide an example below. The main point is that if this is correct, then non-epistemic considerations can play a role in justifying the pursuit of a question.

However, our claim is not merely that non-epistemic values can partly justify the pursuit of some questions, but that they can justify the pursuit of some questions that, if correctly answered, can mitigate transient underdetermination. This has two defining features. First, a correct answer to the question will make previously unavailable epistemic considerations available. ${ }^{2}$ Second, these hitherto unavailable epistemic considerations will break up the gridlock characteristic of transient underdetermination. These two considerations are indices of how "promising" it is to pursue the question. Paradigmatically, an answer to this question promises new evidence that bears on the underdetermined hypotheses. However, owing to our broad notion of epistemic consideration, the question might also suggest new rules of reasoning or methods that have the same effect.

\subsubsection{Long Run $\left(t_{2}\right)$}

Finally, if all goes well, in the third and final stage - the long run - this pursuit makes good on its promise, and scientists have good epistemic reasons to believe one hypothesis over another. Hence, only epistemic considerations figure as rational determinants of belief, even though nonepistemic considerations are sometimes instrumental in pursuing the questions that led to the discoveries of those determinants.

${ }^{2}$ Importantly, these successful pursuits only make previously unknown epistemic considerations available; they do not magically transform non-epistemic considerations into epistemic ones. Whether or not something is an epistemic consideration is conceptually independent of whether it is discovered through successful pursuit. 
To summarize, in the short run $\left(t_{1}\right)$, a set of goals $(G)$ justifies pursuit of a question $(Q)$, the answer to which promises to yield an epistemic consideration $(e)$. If that pursuit is successful in the long run $\left(t_{2}\right)$, then the epistemic consideration provides reasons for believing one hypothesis $\left(h^{*}\right)$ over another $(h)$. We call any member of $G$ that plays this role an inquiry ticket. Thus, our claim is that some inquiry tickets are non-epistemic considerations. We shall now establish this claim with a case study from biology.

\subsection{Case Study in Biology}

Gould's (1978) and Gould and Lewontin's (1979) well-known critiques of Barash's (1976) work on mountain bluebirds helpfully illustrates the way non-epistemic values can function as inquiry tickets. Barash observed two pairs of mountain bluebirds during nest construction and egg-laying and cataloged the birds' response to the presence of a physical model of a male mountain bluebird, placed near the nest at three 10-day intervals. ${ }^{3}$ Barash reports that male bluebirds attacked both their female mates and the model male bird when the model was placed near the nest during construction and before eggs were laid, but did not show aggression toward female mates after eggs were laid. Barash offers an adaptive evolutionary explanation for this pattern in behavior: male bluebirds were concerned about being "cuckolded" by their female mates before eggs were laid, but not after. Let Barash's anti-cuckoldry explanation be our hypothesis $h$. As Lewontin and Gould point out, this hypothesis is underdetermined in the face of a competing explanation that is equally compatible with Barash's observations; namely that the birds may not be demonstrating an "anti-cuckoldry" effect, but may have become accustomed to the presence

${ }^{3}$ Following Barash, we use the word "model" to denote a physical facsimile of a male bluebird. Distinguish this from other meanings of "model" used by philosophers of science. 
of the model or may be behaving this way independently of their concern for the propagation of their genes. Call this competing hypothesis $h^{*}$. If we take Barash's observations as the set of available epistemic considerations $E_{0}$, choosing between $h$ and $h^{*}$ is underdetermined by $E_{0}$ at time $t_{0}$.

Recall that values function as inquiry tickets in relation to a research question, where a correct answer will adjudicate between two competing hypotheses. In this case, the question $Q$ of interest at time $t_{0}$ is presumably something like Gould's suggestion: do mountain bluebirds demonstrate the same behavior toward a model when their first exposure to the model is after eggs are laid?

Gould asserts that non-epistemic political values motivate much of sociobiological inquiry like Barash's work, which he summarizes as "a defense of existing social arrangements as part of our biology" $(1978,532)$. While this is certainly a non-epistemic value, note that any number of other epistemic and non-epistemic values could also have served as inquiry tickets to motivate pursuit of $Q$ in this case. In a later paper, (Morton, Geitgey, and McGrath 1978, 968) pursue exactly this question, and write that they "hoped to confirm that [Barash's] tentative conclusions represented a widespread evolutionary reality." This suggests that values like the fit with background assumptions or anticipated fertility of their experiment lead them to pursue $Q$ as a source of epistemic considerations that would alleviate the transient underdetermination between $h$ and $h^{*}$. In this case, at time $\mathrm{t}_{2}$, their pursuit of $Q$ is successful: Morton et al. demonstrate that, in fact, male bluebirds' behavior toward the introduction of a model male bluebird did not depend on nesting stage (in fact, males in the larger study demonstrated no aggression toward the model at any stage, but female bluebirds repeatedly destroyed it, much to the experimenters' distress). This new evidence $e$, taken together with Barash's original $E_{0}$, better supported Gould's 
hypothesis that the bluebirds' behavior was independent of concerns about gene-propagation, favoring $h^{*}$ over Barash's anti-cuckoldry hypothesis, $h$.

\section{Implications for Science and Values Debates}

Now that we have shown how non-epistemic values can function as inquiry tickets, we situate our approach within the broader literature on science and values. In comparison to other positions in this literature, our view is relatively measured in the role that it accords to nonepistemic values. Even the staunchest "value-freedom fighters" grant that non-epistemic values partially determine which research questions are pursued. Furthermore, our claim is not that every pursuit in response to every case of transient underdetermination is value-laden. Finally, non-epistemic considerations function as inquiry tickets only when adjoined to an epistemic goal-namely to correctly answer a scientific question that will alleviate transient underdetermination through epistemic considerations.

Most strikingly, our view is consistent with (but does not entail) epistemic considerations being the only rational determinants for accepting or rejecting hypotheses. This is the core claim of the much-maligned "value-free ideal." Indeed, it is frequently identified with the value-free ideal (Douglas 2016, Elliott 2017). Following others, we will call this particular aspect of the value-free ideal "impartiality" (Anderson 2004, Lacey 1999). Since inquiry tickets concern pursuit, but not acceptance, impartiality is undisturbed. ${ }^{4}$

\footnotetext{
${ }^{4}$ Section 2's characterization of the long-run framed inquiry tickets in terms of belief. Unlike belief, acceptance is (less controversially thought to be) voluntary, can be rational even when its content is known to be false, and is sensitive to context-specific purposes (Cohen 1992). For
} 
Are we being too tame in aligning ourselves with impartiality? This question suggests two possible answers. First, inquiry tickets may be only one part of a larger story about values in science. Hence, although inquiry tickets are compatible with impartiality, other parts of that story may not be. Alternatively, inquiry tickets' compatibility with impartiality may be a feature rather than a bug. After all, we seem to get the best of both worlds: we give values an interesting role in scientific inquiry and we preserve a central role for epistemic considerations with little fanfare. We explore the latter route, as it seems the more controversial one in the science and values literature. Hence, in what follows, we defend a conditional claim: if you are a value-freedom fighter, then you can recoup much of what your critics claim as both proprietary and beneficial regarding transient underdetermination. In particular, Section 3.1 defends this conditional when the benefit in question involves resolution of the problem that Elliott (2011), (ChoGlueck 2018), and others call the "gap argument." Section 3.2 defends this conditional when the benefit involves the role of inductive risk in scientific inquiry. ${ }^{5}$

\subsection{The Gap Problem}

The value-free ideal is sometimes abandoned so that non-epistemic considerations can settle underdetermination problems without forfeiting scientific rationality. Consider the following inconsistent set:

these reasons, acceptance is far likelier to be "partial," i.e. rationally influenced by non-epistemic considerations. Thus, our arguments that acceptance is impartial suggest, a fortiori, that belief is also impartial.

${ }^{5}$ Our view has some affinities with Elliott and McKaughan's (2009) approach, though unlike us, they do not aim to replicate the strengths of positions opposed to the value-free ideal. 
G1. If non-epistemic considerations can be tiebreakers in cases of transient underdetermination, then scientists may appeal to non-epistemic considerations when accepting or rejecting hypotheses.

G2. Non-epistemic considerations can be tiebreakers in cases of transient underdetermination.

G3. Impartiality: Scientists should only appeal to epistemic considerations when accepting or rejecting hypotheses.

Call the task of rendering this set consistent the Gap Problem. Critics of the value-free ideal frequently accept G1 and G2, and hence deny impartiality (G3). By contrast, since valuefreedom fighters accept impartiality (G3), they take non-epistemic considerations to be ill-suited as rational determinants of acceptance and thereby deny G2 (Haack 1996). As is so often the case, one philosopher's modus ponens is another's modus tollens.

The inquiry ticket account splits the difference by recasting G1 in terms of pursuit:

G1*. If non-epistemic considerations can be tiebreakers in cases of transient underdetermination, then scientists may appeal to non-epistemic considerations when pursuing certain questions.

Clearly, pursuing a question about a theory does not entail accepting that theory. Indeed, one may pursue such a question with some expectation that the answer will disconfirm the theory. Similarly, accepting a theory does not entail that one pursues a question about that theory, as one's curiosity may lie elsewhere. Hence, if non-epistemic values are reasons for pursuit, they need not be reasons for acceptance. To put this another way, non-epistemic considerations only play a central role in the short run, where pursuit is at issue. Non-epistemic values do not play the same role in the long run, when rational acceptance of theories and hypotheses takes center 
stage. Thus, even if one is partial to impartiality, inquiry tickets are effective in recouping some role for non-epistemic values in closing the gap.

\subsection{The Problem of Inductive Risk}

Owing to Douglas' $(2000,2009)$ resuscitation and reinvigoration of (Rudner 1953), inductive risk has become the dominant tool for critics of the value-free ideal. Paradigmatically, inductive risk theorists hold that the benefits of being right and costs of being wrong about a hypothesis determines when there is enough evidence to accept it. Thus, in a certain sense, inductive risk theorists take non-epistemic considerations to determine what counts as sufficient evidence-in our parlance, what counts as an adequate set of epistemic considerations (Biddle 2013, Douglas 2000, Wilholt 2009).

(Biddle 2013) connects the inductive risk framework to transient underdetermination. If inductive risk approaches are correct, then the very idea of basing one's acceptance of a hypothesis on "enough" evidence (epistemic considerations) is already informed by nonepistemic considerations (e.g. the expected value of that assessment). Hence, there is no way to be impartial (in the sense described above) without already undertaking value commitments. Thus, suspending judgment in the face of transient underdetermination may be epistemically irrational if the expected costs of being wrong about the hypothesis greatly outweigh the expected benefits of being right. For instance, agnosticism about a drug's effectiveness may not be a rational response to the evidence if the drug's benefits are modest and its side-effects are lethal.

As with wishful thinking, we can see inductive risk theorists and proponents of impartiality as resolving the following inconsistent set in competing ways: 
IR1. Accepting or rejecting a hypothesis at a given time depends upon whether the epistemic considerations are sufficiently strong.

IR2. Whether the epistemic considerations are sufficiently strong is " a function of the importance, in a typically ethical sense, of making a mistake in accepting or rejecting the hypothesis"' (Rudner, 1953, p. 2, emphasis in original).

IR3. If accepting or rejecting a hypothesis at a given time depends upon the ethical importance of being mistaken in doing so, then scientists should appeal to nonepistemic considerations when accepting or rejecting hypotheses.

IR4. Impartiality: Scientists should only appeal to epistemic considerations when accepting or rejecting hypotheses. ${ }^{6}$

Inductive risk theorists will accept IR1-IR3, but reject IR4. Traditional value-freedom fighters maintain IR1, IR3, and IR4, but reject IR2. Typically, they claim that insofar as IR2 is true, it pertains only to the acceptance characteristic of public policy; IR2 does not pertain to the acceptance characteristic of scientific research (Mitchell 2004).

As with wishful thinking, we defend the conditional claim that if one accepts impartiality, then inquiry tickets recoup many of the attractions of the inductive risk approach. Like traditional value-freedom fighters, we can reject IR2. However, we side with inductive risk theorists in holding that something similar to it applies to scientific research. We deal with this by recasting IR2 in terms of pursuit:

\footnotetext{
${ }^{6}$ This is loosely patterned on (Biddle 2013, 126), who reconstructs and endorses Rudner's argument, though we have recast it so as to make its challenge to impartiality more explicit.
} 
IR2*. Whether the epistemic considerations are sufficiently strong is a function of the importance, in a typically ethical sense, of being misguided in one's pursuits. Pursuits can be ethically misguided in two ways. First, one may fail to pursue a question one ought to pursue given the ethical risks involved (roughly: a failure of due diligence). Second, one may pursue a question one ought not to pursue because allocating resources to this pursuit has ethically harmful opportunity costs or pernicious social effects. ${ }^{7}$

On the picture being sketched here, inductive risk's non-epistemic dimensions may affect pursuit (IR2*), without (directly) affecting acceptance (IR4). The resulting picture is plausible. When people take excessive risks, it is commonplace to wonder what questions they have asked themselves. Typically, these questions will be of the form "What would happen if I did X?" We then expect them to search out the answers to these questions if doing $\mathrm{X}$ has important ethical consequences. In other words, we expect them to pursue these questions. Science is no exception. For instance, in testing a drug's effectiveness, it would be reasonable to demand that biomedical researchers pursued questions about harmful side effects. Furthermore, pursuit need not bear on acceptance, as already noted in Section 3.1. In other words, it appears possible for scientists to be rational in relying solely on epistemic considerations to set relevant evidential thresholds in accepting that a drug has a certain effect, while having ethical obligations to pursue

${ }^{7}$ The idea of misguided pursuits neutralizes a potential counterexample, in which ideologically skewed scientific communities' pursuits only yield epistemic considerations favoring one hypothesis. Such scientists may be misguided in failing to pursue different questions motivated by alternative values. However, they need not be irrational in accepting the hypothesis favored by the available epistemic considerations, as impartiality requires. 
further questions about its side-effects' harmfulness before communicating these results to the broader public. This seems to replicate much of what inductive risk theorists seek, yet it is compatible with impartiality as a scientific ideal.

Of course, our account holds that scientists should update their acceptance once the questions that they have pursued are answered. Again, this underscores the importance of the temporal dimension of values. Since the available epistemic considerations differ from the short run to the long run, one's acceptance should also change at these different stages in inquiry.

\section{Conclusion}

We have introduced the concept of inquiry tickets, and argued that they are a useful way of framing how scientists overcome transient underdetermination. Using an example from biology, we argued that some non-epistemic considerations are inquiry tickets. Finally, we have shown that if one is sympathetic to the ideal of value-free science, then inquiry tickets are a useful way to recognize some of the same insights as those who reject this ideal.

We are optimistic that inquiry tickets provide a novel and fruitful way of developing the science and values literature in general, and of defending impartiality in particular. For instance, not all issues about science and values are tethered to underdetermination. Consequently, these issues may point to other philosophical benefits that conflict with the value-free ideal. To engage these challenges in the future, we note that our account can be generalized beyond cases of transient underdetermination. We would simply take the framework in Section 2.1 and omit its first stage, where transient underdetermination occurs, while slightly revising the last two stages to reflect this change: 
Short Run: At time $t_{1}$, the set of available epistemic considerations is $E_{0}$, and pursuing a question $Q$ is conducive to a set of goals $G$ adopted by some scientist $S$.

Long Run: At time $t_{2}$, the pursuit of $Q$ succeeds. As a result, $e$ is available, and $h$ is better supported by the conjunction of $e$ and the members of $E_{0}$ than it was at $t_{1}$. Hence, it is more rational to believe $h$ at $t_{2}$ than it was to believe $h$ at $t_{1}$.

In future work, we hope to ascertain whether this more general approach to inquiry tickets can replicate further benefits that critics of the value-free ideal claim as their own.

Additionally, there are further challenges to the value-free ideal. These include the "descriptive challenge" that the value-free ideal is not faithful to many instances of good scientific practice, and the "boundary challenge" that epistemic considerations cannot be neatly separated from non-epistemic considerations (Douglas 2016). ${ }^{8}$ While space prohibits addressing those challenges here, we speculate that further and more detailed case studies might address the descriptive challenge, and that the distinctions between the short and the long run, and among pursuit and other cognitive attitudes (e.g. acceptance), form the basis of a defensible boundary between the non-epistemic and the epistemic.

\footnotetext{
${ }^{8}$ Section 3.2 suggests an answer to Douglas' third and final, "normative" challenge to the valuefree ideal.
} 


\section{References}

\section{!!! INVALID CITATION !!! .}

Anderson, Elizabeth. 1995. "Knowledge, Human Interests, and Objectivity in Feminist Epistemology." Philosophical Topics 23 (2):27-58.

Anderson, Elizabeth. 2004. "Uses of Value Judgments in Science: A General Argument, with Lessons from a Case Study of Feminist Research on Divorce." Hypatia 19 (1):1-24. doi: 10.1111/j.1527-2001.2004.tb01266.x.

Barash, David P. 1976. "Male Response to Apparent Female Adultery in the Mountain Bluebird (Sialia currucoides): An Evolutionary Interpretation." The American Naturalist 110 (976):1097-1101.

Biddle, Justin. 2013. "State of the field: Transient underdetermination and values in science." Studies in History and Philosophy of Science Part A 44 (1):124-133. doi: 10.1016/j.shpsa.2012.09.003.

Brown, Matthew J. 2013. "Values in Science beyond Underdetermination and Inductive Risk." Philosophy of Science 80 (5):829-839. doi: 10.1086/673720.

ChoGlueck, Christopher. 2018. "The Error Is in the Gap: Synthesizing Accounts for Societal Values in Science." Philosophy of Science 85 (4):704-725. doi: 10.1086/699191.

Cohen, L. Jonathan. 1992. An essay on belief and acceptance. Oxford: Clarendon Press. 
Douglas, Heather E. 2000. "Inductive risk and values in science." Philosophy of Science 67 (4):559-579.

Douglas, Heather E. 2009. Science, policy, and the value-free ideal. Pittsburgh: University of Pittsburgh Press.

Douglas, Heather E. 2016. "Values in Science." In The Oxford Handbook of Philosophy of Science, edited by Paul Humphreys, 609-630. Oxford: Oxford University Press.

Duhem, Pierre. 1954. The aim and structure of physical theory. Translated by Philip P. Wiener. 2nd ed. Princeton, N.J.: Princeton University Press. Original edition, 1914.

Elliott, Kevin C. 2011. Is a little pollution good for you? : incorporating societal values in environmental research, Environmental ethics and science policy series. New York: Oxford University Press.

Elliott, Kevin C. 2017. A tapestry of values : an introduction to values in science. New York, NY: Oxford University Press.

Elliott, Kevin C., and Daniel J. McKaughan. 2009. "How Values in Scientific Discovery and Pursuit Alter Theory Appraisal." Philosophy of Science 76 (5):598-611. doi: $10.1086 / 605807$.

Gould, Stephen Jay. 1978. "Sociobiology: the art of storytelling." New Scientist 80 (1129):53033.

Gould, Stephen Jay, and Richard C Lewontin. 1979. "The spandrels of San Marco and the Panglossian paradigm: a critique of the adaptationist programme." Proceedings of the Royal Society of London.

Haack, Susan. 1996. "Science as Social? - Yes and No." In Feminism, Science, and the Philosophy of Science, edited by Lynn Hankinson Nelson and Jack Nelson, 79-93. Dordrecht: Springer Netherlands.

Intemann, Kristen. 2005. "Feminism, underdetermination, and values in science." Philosophy of Science 72 (5):1001-1012.

Kuhn, Thomas S. 1977. "Objectivity, value judgement, and theory choice." In The essential tension, 320-339. Chicago: University of Chicago Press.

Lacey, Hugh. 1999. Is science value free? : values and scientific understanding. London ; New York: Routledge.

Laudan, Larry. 1977. Progress and its problems : toward a theory of scientific growth. Berkeley: University of California Press.

Longino, Helen E. 1990. Science as social knowledge: values and objectivity in scientific inquiry. Princeton, N.J.: Princeton University Press.

Longino, Helen E. 2002. The fate of knowledge. Princeton, N.J.: Princeton University Press.

Mitchell, Sandra D. 2004. "The Prescribed and Proscribed Values in Science Policy." In Science, Values, and Objectivity, edited by Peter Machamer and Gereon Wolters, 245-255. Pittsburgh, PA: University of Pittsburgh Press.

Morton, Eugene S., Mary S. Geitgey, and Susan McGrath. 1978. "On Bluebird 'Responses to Apparent Female Adultery'." The American Naturalist 112 (987):968-971.

Nelson, Lynn Hankinson. 1990. Who knows : from Quine to a feminist empiricism. Philadelphia: Temple University Press.

Nelson, Lynn Hankinson. 1993. "Epistemological communities." In Feminist Epistemologies, edited by Linda Alcoff and Elizabeth Potter, 121-160. London: Routledge. 
Quine, W. V. 1951. "Main Trends in Recent Philosophy: Two Dogmas of Empiricism." The Philosophical Review:20-43.

Rudner, Richard. 1953. "The Scientist Qua Scientist Makes Value Judgments." Philosophy of Science 20 (1):1-6. doi: 10.2307/185617.

Seselja, Dunja, Laszlo Kosolosky, and Christian Strasser. 2012. "The rationality of scientific reasoning in the context of pursuit: Drawing appropriate distinctions." Philosophica 86:51-82.

Whitt, Laurie Anne. 1990. "Theory Pursuit: Between Discovery and Acceptance." Proceedings of the Biennial Meetings of the Philosophy of Science Association 1:467-483.

Wilholt, Torsten. 2009. "Bias and values in scientific research." Studies In History and Philosophy of Science Part A 40 (1):92-101. 
